\title{
Increasing outpatient treatment of mild community-acquired pneumonia: systematic review and meta-analysis
}

\author{
J.D. Chalmers, A.R. Akram and A.T. Hill
}

ABSTRACT: In order to identify, synthesise and interpret the evidence relating to strategies to increase the proportion of low-risk patients with community-acquired pneumonia treated in the community, we conducted a systematic review of intervention studies conducted between 19812010.

Articles were included if they compared strategies to increase outpatient care with usual care. Outcomes were: the proportion of patients treated as outpatients, mortality, hospital readmissions, health related quality of life, return to usual activities and patient satisfaction with care.

The main analysis included six studies. The interventions in these studies were generally complex, but all involved the use of a severity score to identify low-risk patients. Overall, a significantly larger numbers of patients were treated in the community with these interventions (OR 2.31, 95\% CI 2.03-2.63). The interventions appear safe, with no significant differences in mortality (OR $0.83,95 \% \mathrm{Cl} 0.59-1.17$ ), hospital readmissions (OR $1.08,95 \% \mathrm{Cl} 0.82-1.42$ ) or patient satisfaction with care (OR 1.21, 95\% $\mathrm{Cl} 0.97-1.49)$ between the intervention and control groups. There was insufficient data regarding quality of life or return to usual activities. All studies had significant limitations.

The available evidence suggests that interventions to increase the proportion of patients treated in the community are safe, effective and acceptable to patients.

KEYWORDS: Acute respiratory infection, community-acquired pneumonia, disease management, guidelines, guidelines for management of pneumonia, infections

ommunity-acquired pneumonia (CAP) is the most common infectious disease presenting to emergency departments in western countries [1]. Population-based studies of CAP in Europe suggest an incidence of CAP requiring hospitalisation of $1.98-2.6$ per 1,000 population per year $[2,3]$. Approximately $75 \%$ of cases are managed in the community, where mortality is very low. The mortality rate in hospitalised patients is reported to be $5-15 \%[4,5]$.

Treatment of CAP costs over 8 billion dollars annually in the USA [6]. More than $90 \%$ of that expenditure relates to the cost of in-patient care [6]. The initial decision, made by the attending physician, to admit the patient to hospital or discharge the patient from hospital is, therefore, crucial.

In recent years, severity assessment tools such as the Pneumonia Severity Index (PSI) and the
CURB65 (new onset mental confusion, urea $>7 \mathrm{mM}$, respiratory frequency $\geqslant 30$ breaths $\cdot \mathrm{min}^{-1}$, systolic blood pressure $<90 \mathrm{mmHg}$ or diastolic blood pressure $\leqslant 60 \mathrm{mmHg}$, and age $\geqslant 65 \mathrm{yrs}$ ) score have been developed $[4,5]$. These tools allow patients to be categorised into groups at low, intermediate and high risk for 30-day mortality. Patients at low risk for mortality are more likely to be suitable for outpatient care. These tools are now promoted by almost all national and international guidelines to aid clinicians in making the site-ofcare decision [1].

Strategies to increase the proportion of patients treated in the community have the potential to significantly decrease hospital costs, but must be safe and acceptable to patients [7].

In this study, we systematically reviewed the published literature to identify, synthesise and

Earn CME accreditation by answering questions about this article. You will find these at the back of the printed copy of this issue or online at www.erj.ersjournals.com/misc/cmeinfo.xhtml

AFFILIATIONS

Royal Infirmary of Edinburgh, Edinburgh, UK.

CORRESPONDENCE

J.D. Chalmers

Dept of Respiratory Medicine Royal Infirmary of Edinburgh 51 Little France Crescent Old Dalkeith Road Edinburgh EH16 4SA E-mail: jamesdchalmers@ googlemail.com

Received:

April 272010 Accepted after revision: Aug 012010 First published online: Aug 202010 
interpret the evidence relating to strategies to increase the proportion of low-risk patients with CAP treated in the community.

The aim of the study was to establish if these interventions could increase the proportion of patients treated in the community without compromising patient satisfaction with care, healthyrelated quality of life and return to usual activities, or increasing hospital readmissions or mortality.

\section{METHODS}

This was a systematic review and meta-analysis conducted and reported according to Preferred Reporting Items for Systematic Reviews and Meta-Analyses (PRISMA) recommendations [8].

\section{Search strategy}

The present review was based on a search of PubMed and EMBASE for articles using keywords "outpatient", "discharge", "home", "hospitalisation/hospitalization" or "guideline" in combination with "community-acquired pneumonia" was performed between January 1981 and April 2010. Nonrelevant studies were excluded based on title and abstract review only. Full articles of all potentially appropriate abstracts were retrieved and reviewed by investigators. No language restriction was applied. Only peer-reviewed data was included: therefore, conference abstracts were excluded. The search strategy was supplemented by reviewing of reference lists, bibliographies and the investigators' files.

\section{Study inclusion criteria and quality assessment}

Data was independently extracted from each relevant study by two abstractors and these abstractors carried out quality assessment using standardised criteria [9]. Quality assessments were performed separately and disagreements resolved by a third independent abstractor.

Studies were included if they met the following criteria: 1) original publication; 2) describe an intervention aimed to increase the proportion of patients treated in the community; 3 ) include a control group in which the intervention was withheld; and 4) include data reporting the safety of the intervention.

Studies reporting outpatient care but without control data were not included.

\section{End-points}

The primary outcome was the proportion of patients treated in the community in the intervention groups compared to the control groups. Measures of safety were also assessed as follows: mortality, readmission to hospital in communitytreated patients, patient satisfaction with care, health-related quality of life, and return to work or usual activities.

\section{Statistical analysis}

Statistical analysis was performed using Review Manager, version 5 (Cochrane IMS, Oxford, UK). Pooled data are presented as OR (95\% CI). A fixed-effects model was used to pool the results of individual studies. Heterogeneity of study results were assessed by calculating a Chi-squared test of heterogeneity and the $\mathrm{I}^{2}$-test for inconsistency. Significant heterogeneity was predefined as a Chi-squared test $\mathrm{p}<0.1$ or an $\mathrm{I}^{2}$-test $>50 \%$. Publication bias was assessed using the Funnel plot method.

\section{RESULTS}

The literature review identified six studies for inclusion in the meta-analysis [10-15]. Details of the literature review are shown in figure 1.

Details of each study are shown in table 1 .

The interventions used in each study were generally complex, but all included a scoring system to identify low-risk patients. In five studies, the PSI was used to help determine where patients should be treated in the intervention arm. In one study, the authors derived their own criteria for in-patient care and then implemented this. No clinical trials using any of the other available severity scores were identified.

\section{Description of included studies}

In the study by ATLAs et al. [10], a single-centre intervention, the authors implemented a practice guideline for patients with low PSI scores. The study included patients with PSI scores IIII and excluded patients with significant hypoxaemia, patients with immunosuppression, injecting drug users and patients with other co-morbidities that were considered a contraindication to outpatient care. The intervention involved promoting the use of the PSI in the emergency department and supporting discharge by providing nursing visits at home, standardised antibiotic treatment (clarithromycin monotherapy) and access to a primary care physician. Care after the intervention was compared to a retrospective control cohort identified from case-note review. Significantly more patients were treated as outpatient in the intervention compared with the control cohort [10].

In the study by MARRIE et al. [11], the intervention was the implementation of the critical pathway composed of three parts: 1) promoting the use of the PSI; 2) treatment with levofloxacin; and 3) implementation of a practice guideline that included standard microbiological tests, i.v.-to-oral switch criteria and hospital discharge criteria. Nine hospitals implemented this critical pathway while 10 hospitals managed patients according to their usual practice. This did not include pneumonia severity scoring or the other components of the critical pathway [11].

In the study by DEAN et al. [12], a clinical practice guideline based on the American Thoracic Society guidelines was

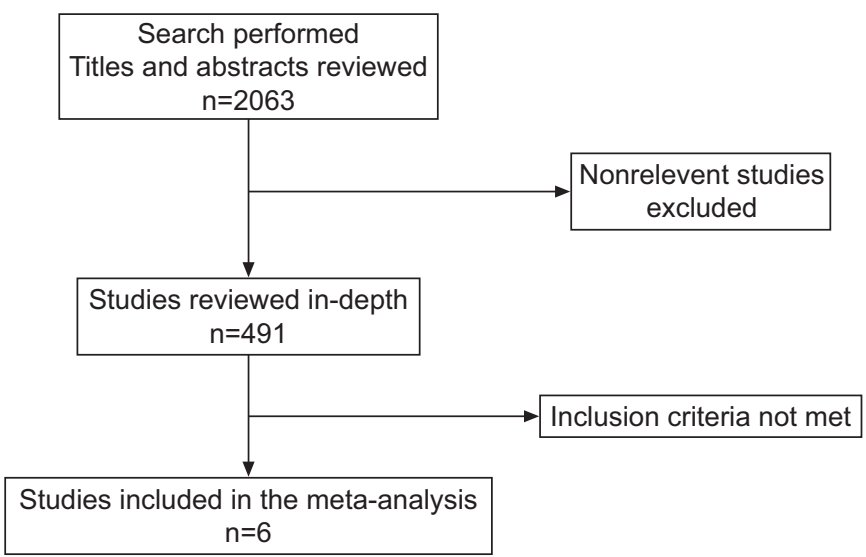

FIGURE 1. Process of literature review. 


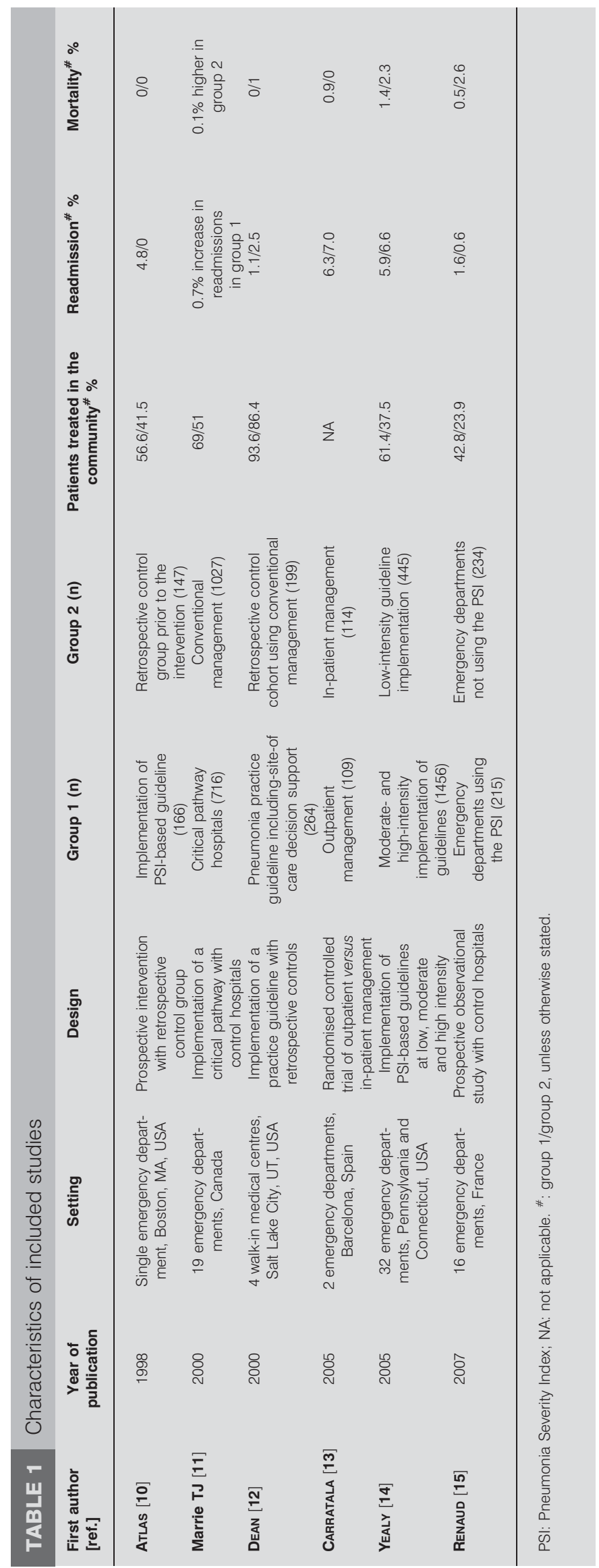

introduced. The guideline included a decision support system to determine site of care that included a scoring system. This study is unique in not using the PSI to determine initial site of care; instead, the authors determined their own criteria for outpatient care and incorporated these into the guideline. The study is also unique as it took place in community-based walkin medical centres rather than emergency departments, as was the case in the other studies. Data for 12 months after the introduction of the guideline were compared to a retrospective control group of patients treated before implementation of the guideline [12].

In the study by CARRATALA et al. [13], patients were randomised to out- versus in-patient management. Outpatients were treated with oral levofloxacin and in-patients treated with sequential i.v. then oral levofloxacin. Patients were excluded if they were intolerant of quinolones, were pregnant or breast feeding, or had respiratory failure, unstable comorbidities, pneumonia complications (pleural effusion or lung abscess), or were unable to take oral medications [13]. Outcomes were determined 30 days after randomisation and included mortality, the number of readmissions, and adverse drug reactions and patient satisfaction with care.

YEALY et al. [14] conducted as cluster-randomised trial at 32 emergency departments in Pennsylvania and Connecticut, USA. Centres were randomised to one of three intensities of pneumonia guideline implementation. The intervention included instructions to use the PSI, and to manage patients in class I-III without oxygen desaturation as outpatients. The guideline also suggested administration of antibiotics for inpatients within $4 \mathrm{~h}$ and recommended appropriate empirical antibiotic therapy. The low-intensity strategy simply involved writing to medical directors of hospitals suggesting that they develop pneumonia quality improvement strategies and mailing the emergency department with the guideline. The moderate- and high-intensity strategies included the measures for the low-intensity group but also an on-site teaching session on how to use the PSI and encouragement of outpatient treatment. The high-intensity group also included a number of additional reminders and feedback systems [14].

In the study by RENAUD et al. [15], an observational study, eight emergency departments in which the PSI was used to determine site of care were compared with eight emergency departments in which the PSI was not used. PSI-using hospitals were provided with posters and pocket cards reminding them to use the PSI, while control hospitals were not. No other interventions were used. Each hospital completed data collection for 3-5 months and the primary outcome was the proportion of patients discharged from the emergency department [15].

The results of these studies are summarised in table 1.

\section{Outpatient management using clinical guidelines}

We included five studies in the meta-analysis for outpatient care. The study by CARRATALA et al. [13] was unique in randomising all patients to out- or in-patient treatment rather than implementing a clinical guideline to increase the proportion of patients treated in the community; therefore, this was not included in this part of the analysis. The definition of outpatient treatment was only specifically given 
in the study by YEALY et al. [14], defined as discharge from the emergency department to the community within $24 \mathrm{~h}$. In the other studies, outpatient treatment was assumed to meet the same definition.

The analysis included a total of 2,817 patients in the intervention groups and 2,052 patients in the control groups. In raw analysis, $64.6 \%$ of patients in the intervention group were treated in the community compared with $48.7 \%$ of patients in the control groups.

In the meta-analysis, the interventions were associated with a significant increase in outpatient-managed patients (OR 2.31, $95 \%$ CI 2.03-2.63). The Forest plot is shown in figure 2. There was no significant heterogeneity.

\section{Safety of the intervention}

For the two measures of safety, mortality was not increased (OR 0.83, 0.59-1.17) (fig. 3).

For hospital readmissions, the studies by AtLAs et al. [10], DEAN et al. [12], YEALY et al. [14] and RENAUd et al. [15] reported readmissions only for patients initially treated in the community in both intervention and control groups. The definition of readmission was unclear in the study by MARRIE et al. [11]. CARRATAla et al. [13] compared readmissions in patients discharged from the emergency department to those patients initially hospitalised and then subsequently discharged.

Separately, none of these studies showed an increase in hospital readmissions. Similarly, the pooled analysis showed no increase in hospital readmissions (OR 1.08, 95\% CI 0.82 1.42; fig. 4). There was no significant heterogeneity in these analyses.

\section{Patient satisfaction with care}

Only three studies reported data for patient satisfaction with care between intervention and control groups. In the studies by CARRATALA et al. [13] and ATLAs et al. [10], patients were asked to rate their satisfaction with care at 4 weeks on a scale of 1-5 (very unsatisfactory to very satisfactory) with 4 or 5 considered satisfied. In the study by YEALY et al. [14], telephone interviews were conducted at day 30 and patients were asked if they were satisfied with their initial site of care, their emergency department care and their overall medical care.

Pooling the results of the three studies, there was no difference between the intervention and control groups (OR 1.21, 95\% CI $0.97-1.49 ; \mathrm{p}=0.09)$ in the proportion of patients reporting satisfaction with overall care. There was no significant heterogeneity in the analysis (fig. 5). This conclusion was based predominantly on the results of the study by YEALY et al. [14], which was significantly larger than the other two studies.

\section{Return to usual activities and quality of life}

There were insufficient data to pool studies of return to usual activities or quality of life. ATLAS et al. [10] reported return to daily activities, with $92 \%$ of patients in the intervention and $85 \%$ in the retrospective control group reporting return to usual activities $(\mathrm{p}>0.05)$. They also reported no difference in patients reporting general health excellent or very good at 4 weeks.

The study by MARRIE et al. [11] reported quality of life using the Short-Form 36 physicial component summary scale (SF-36) and reported no significant difference between intervention and control hospitals. CARRATALA et al. [13] also assessed healthrelated quality of life using the SF-36 tool and found no significant difference at day 7 or day 30 in health-related quality of life between patients managed as in-patients or outpatients. YEALY et al. [14] assessed return to work and usual activities at day 30 and found no significant differences in these parameters between the low-, moderate- and high-intensity guideline implementation groups. Finally, RenAud et al. [15] and DEAN et al. [12] did not assess return to usual activities or quality of life.

\section{Publication bias}

In each of the analyses, inspection of funnel plots did not suggest any evidence of publication bias (data not shown).

\section{Quality assessment}

Each of the included studies had significant limitations. The studies by ATLAS et al. [10] and DEAN et al. [12] utilised a retrospective control cohort design, a method associated with a significant risk of bias. Similarly, the centres included in the study by RENAUD et al. [15] were not randomised. Instead, the study included hospitals that had decided independently to

\begin{tabular}{|c|c|c|c|c|c|c|c|c|c|c|}
\hline \multirow[t]{2}{*}{ First author [ref.] } & \multicolumn{2}{|c|}{ Intervention } & \multicolumn{2}{|c|}{ Control } & \multirow[t]{2}{*}{ Weight $\%$} & \multirow[t]{2}{*}{ OR $(95 \% \mathrm{Cl})$} & \multirow[t]{2}{*}{ Year } & \multirow[t]{2}{*}{ Favours control } & \multicolumn{2}{|c|}{ Favours intervention } \\
\hline & Events $\mathrm{n}$ & Total $\mathbf{n}$ & Events $\mathrm{n}$ & $\overline{\text { Total } n}$ & & & & & & \\
\hline AtLAs [10] & 94 & 166 & 61 & 147 & 9.2 & $1.84(1.17-2.88)$ & 1998 & & $\longrightarrow$ & \\
\hline MARRIE [11] & 494 & 716 & 524 & 1027 & 44.0 & $2.14(1.75-2.61)$ & 2000 & & & \\
\hline Yealy [14] & 894 & 1456 & 167 & 445 & 32.5 & $2.65(2.13-3.30)$ & 2005 & & & \\
\hline Renaud [15] & 92 & 215 & 56 & 234 & 10.1 & $2.38(1.59-3.56)$ & 2007 & & & \\
\hline Total & 1821 & 2817 & 980 & 2052 & 100.0 & $2.31(2.03-2.63)$ & & & & \\
\hline Heterogeneity: $\mathrm{C}$ & uared $=3$ & $\mathrm{df}=4$ & $(p=0.54)$ & $\%$ & & & & $0.1 \quad 0.2$ & 1 & 10 \\
\hline Test for overall effe & $\mathrm{t}: \mathrm{Z}=12.62$ & $(p<0.000$ & & & & & & & ds ratio & \\
\hline
\end{tabular}

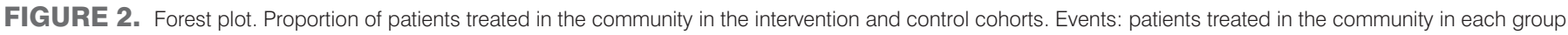
Odds ratio from Maentel-Hentzel fixed-effects model. Whiskers represent 95\% confidence interval. df: degrees of freedom. 


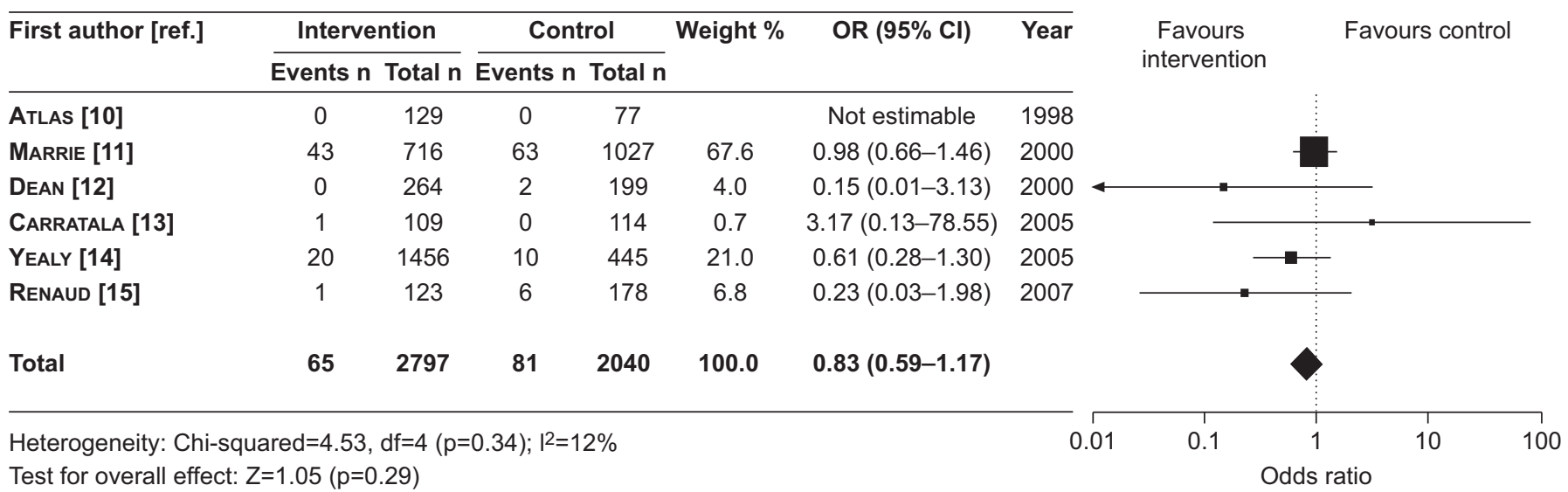

FIGURE 3. Forest plot. Mortality among low-risk patients in the intervention and control cohorts following implementation of measures to increase outpatient management. Events: deaths in each group. Odds ratio from Maentel-Hentzel fixed-effects model. Whiskers represent 95\% confidence interval. df: degrees of freedom.

implement the PSI and control hospitals that had not. There is no way of knowing to what extent other aspects of CAP management were different in these centres, or that the PSI was not used in the control hospitals. The cluster randomisation design utilised in the studies by MARRIE et al. [11] and YEALY et al. [14] are more robust; however, as the PSI is a well known and widely used instrument, randomisation at the hospital level cannot ensure the intended practice at the individual-physician level. The study by CARRATALA et al. [13] was more robust, as a randomised controlled trial in two centres but was underpowered to detect mortality, which is rare in low-risk patients. The study also had to exclude a large proportion of patients as many otherwise low-risk patients are not suitable for outpatient care. All of the guideline interventions were composed of multiple components and, therefore, evaluating which of these components were responsible for the effects seen is not straightforward.

\section{DISCUSSION}

This systematic review and meta-analysis demonstrates that, based on the available evidence, strategies to increase the proportion of patients treated in the community are safe, effective and acceptable to patients. Increased use of outpatient care for low-risk patients, primarily defined using the PSI, was associated with no significant increase in hospital readmissions, patient mortality or patient dissatisfaction with care [10-15]. There were limited data on other safety outcomes, such as health-related quality of life and return to usual activities.

Each of the six included studies had significant limitations and each used a variety of different methods to encourage outpatient management. All of these studies, however, were based on the principle that patients at low risk of death and without important contraindications to outpatient treatment, such as inability to take oral medication or unstable comorbidities, can be treated safely at home. The studies were conducted in the USA, Canada, France and Spain, suggesting that these results can be generalised to different healthcare systems [10-15].

The PSI was developed in 1997 with the aim of identifying a population of patients at low risk for mortality who may be suitable for outpatient therapy [4]. This score has been shown to be reliable in a large number of validation studies [16-20]. Evidence suggests that since the introduction of PSI, the length of stay for patients with CAP may have decreased and more patients may be initially treated in the community [21]. There is,

\begin{tabular}{|c|c|c|c|c|c|c|c|}
\hline \multirow[t]{2}{*}{ First author [ref.] } & \multicolumn{2}{|c|}{ Intervention } & \multicolumn{2}{|c|}{ Control } & \multirow[t]{2}{*}{ Weight $\%$} & \multirow[t]{2}{*}{ OR (95\% Cl) } & \multirow[t]{2}{*}{ Year } \\
\hline & Events $\mathrm{n}$ & Total $n$ & Events $\mathrm{n}$ & Total $\mathrm{n}$ & & & \\
\hline AtLAs [10] & 8 & 129 & 0 & 77 & 0.6 & $10.84(0.62-190.55)$ & 1998 \\
\hline MARRIE [11] & 70 & 716 & 92 & 1027 & 68.1 & $1.10(0.79-1.53)$ & 2000 \\
\hline DeAn [12] & 3 & 244 & 5 & 172 & 5.8 & $0.42(0.10-1.76)$ & 2000 \\
\hline CarRatala [13] & 7 & 110 & 8 & 114 & 7.3 & $0.90(0.32-2.57)$ & 2005 \\
\hline Yealy [14] & 53 & 894 & 11 & 167 & 17.4 & $0.89(0.46-1.75)$ & 2005 \\
\hline Renaud [15] & 2 & 123 & 1 & 178 & 0.8 & $2.93(0.26-32.62)$ & 2007 \\
\hline Total & 143 & 2216 & 117 & 1735 & 100.0 & $1.08(0.82-1.42)$ & \\
\hline
\end{tabular}

Heterogeneity: Chi-squared=5.26, $d f=5(p=0.38) ;\left.\right|^{2}=5 \%$

Test for overall effect: $Z=0.57(p=0.57)$

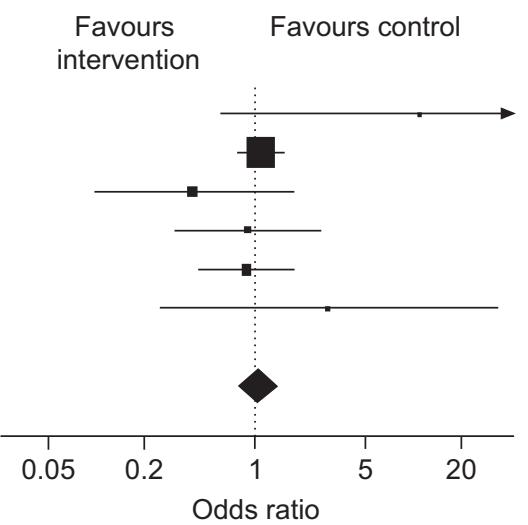

FIGURE 4. Forest plot. Readmissions following hospital discharge in the intervention and control cohorts. Events: readmissions following hospital discharge. Odds ratio from Maentel-Hentzel fixed-effects model. Whiskers represent 95\% confidence interval. df: degrees of freedom. 


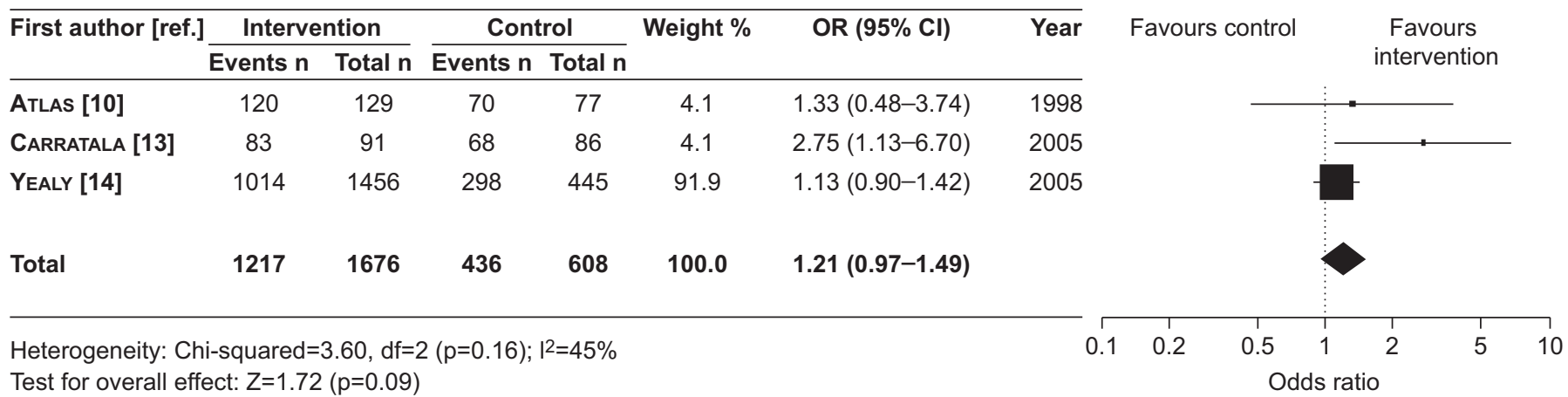

FIGURE 5. Forest plot. Satisfaction with care in the intervention and control cohorts following implementation of measures to increase outpatient management. Events: patients reporting satisfaction with care. Odds ratio from Maentel-Hentzel fixed-effects model. Whiskers represent $95 \%$ confidence interval. df: degrees of freedom.

however, evidence from a number of studies, including those included in this meta-analysis, that a larger proportion of patients could be safely treated in the community [10-15].

Outpatient management has several potential advantages. More than $90 \%$ of hospital costs are associated with in-patient care and even small increases in the proportion of patients treated at home can result in large economic savings [6, 22]. Hospital-acquired infections, such as methicillin-resistant Staphylococcus aureus and Clostridium difficile, are an increasing problem in the UK and internationally $[23,24]$. Reducing the proportion of patients treated in hospital will reduce the risk of patients developing these hospital complications.

\section{Barriers to outpatient treatment}

All of the quality improvement studies found a proportion of low-risk patients still requiring inpatient treatment. Physician judgement is critical in the implementation of severity scores [25]. A number of studies have investigated reasons why lowrisk patients require hospitalisation and show that comorbid illnesses, inability to take oral medications, severity not adequately captured by the PSI and hypoxaemia are frequent in low-risk patients $[25,26]$. These factors must be taken into consideration when implementing outpatient management strategies.

Evidence suggests that physicians overestimate the severity of low-risk patients [26]. It is notable that each of the successful quality improvement studies in this meta-analysis included some degree of physician education and feedback. This is well demonstrated in the study by YEALY et al. [14], in which lowintensity implementation of guidelines, in which physicians were simply provided with the guidelines, were significantly less effective than the moderate-/high-intensity group, in which guidelines were supplemented with physician education and feedback. There is evidence that the PSI and other severity assessment tools are under-utilised in some centres [27].

This study did not identify any clinical studies or trials of severity scores other than the PSI and a site-specific score developed by DEAN et al. [12] to increase the proportion of patients treated in the community. It is uncertain if the results of this meta-analysis can be generalised to other methods or scoring systems [5, 28, 29].

\section{Limitations of the included studies}

As discussed above, potential bias must be considered in interpreting these results. Although the meta-analysis found no indication of publication bias, the methodology of each study varied substantially. The studies by ATLAs et al. [10] and DEAN et al. [12] used retrospective control cohorts. When different methodologies are used to collect data for the intervention and control groups, the risk of bias is increased. The analysis of patient satisfaction with care was largely based on a single study (YeALY et al. [14]) and, therefore, further studies on this end-point would be desirable. This metaanalysis was an aggregate meta-analysis rather than an individual patient data-level analysis. The latter offers several advantages [30] and the use of aggregate methods in this study is a limitation. The pooled results should be treated with caution as, although there was no statistical heterogeneity, there were differences between studies in the interventions used and the characteristics of the healthcare systems in which they were implemented.

\section{Implementation and future studies}

The results of this meta-analysis suggest that emergency departments should be encouraged to develop strategies to manage more patients in the community, using validated criteria to ensure these interventions are safe. Further interventional studies are required, particularly with respect to severity criteria other than the PSI that have not yet been tested for guiding outpatient management. Limited data are available for important end-points, such as health-related quality of life, symptom resolution, and return to work and usual activities, and further studies in this area are needed.

\section{Conclusion}

Current evidence suggests that strategies to increase the proportion of patients treated in the community are safe, effective and acceptable to patients.

\section{SUPPORT STATEMENT}

J.D. Chalmers was supported by a clinical research training fellowship from the Medical Research Council (UK).

\section{STATEMENT OF INTEREST}

None declared. 


\section{REFERENCES}

1 Lim WS, Baudouin SV, George RC, et al. BTS guidelines for the management of community acquired pneumonia in adults: update 2009. Thorax 2009; 64: Suppl. 3, iii1-iii5.

2 Trotter CL, Stuart JM, George G, et al. Increasing hospital admissions for pneumonia, England. Emerg Infect Dis 2008; 14: 727-733.

3 Almirall J, Bolibar I, Vidal J, et al. Epidemiology of communityacquired pneumonia in adults: a population-based study. Eur Respir J 2000; 15: 757-763.

4 Fine MJ, Auble TE, Yealy DM, et al. A prediction rule to identify low-risk patients with community-acquired pneumonia. $N$ Engl J Med 1997; 336: 243-250.

5 Lim WS, van der Eerden MM, Laing R, et al. Defining community acquired pneumonia severity on presentation to hospital: an international derivation and validation study. Thorax 2003; 58: 377-382.

6 Raut M, Schein J, Mody S, et al. Estimating the economic impact of a half-day reduction in length of hospital stay among patients with community-acquired pneumonia in the US. Curr Med Res Opin 2009; 25: 2151-2157.

7 Fine MJ, Pratt HM, Obrosky DS, et al. Relation between length of hospital stay and costs of care for patients with communityacquired pneumonia. Am J Med 2000; 109: 378-385.

8 Liberati A, Altman DG, Tetziaff J, et al. The PRISMA statement for reporting systematic reviews and meta-analyses of studies that evaluate healthcare interventions: explanation and elaboration. Ann Intern Med 2009; 151: W65-W94.

9 Higgins JPT, Green S, eds. Cochrane Handbook for Systematic Reviews of Interventions. Version 5.0.2. Oxford, Cochrane Collaboration, 2009.

10 Atlas SJ, Benzer TL, Borowsky LH, et al. Safely increasing the proportion of patients with community-acquired pneumonia treated as outpatients: an interventional trial. Arch Intern Med 1998; 158: 1350-1356.

11 Marrie TJ, Lau CY, Wheeler SL, et al. A controlled trial of a critical pathway for treatment of community-acquired pneumonia. JAMA 2000; 283: 749-755.

12 Dean NC, Suchyta MR, Bateman KA, et al. Implementation of admission decision support for community-acquired pneumonia. Chest 2000; 117: 1368-1377.

13 Carratala J, Fernandez-Sabe N, Ortega L, et al. Outpatient care compared with hospitalization for community-acquired pneumonia: a randomized trial in low risk patients. Ann Intern Med 2005; 142: 165-172.

14 Yealy DM, Auble TE, Stone RA, et al. Effect of increasing the intensity of implementing pneumonia guidelines: a randomised controlled trial. Ann Intern Med 2005; 143: 881-894.

15 Renaud B, Coma E, Labarere J, et al. Routine use of the Pneumonia Severity Index for guiding the site-of-treatment decision of patients with pneumonia in the emergency department: a multicenter, prospective, observational, controlled cohort study. Clin Infect Dis 2007; 44: 41-49.

16 Buising KL, Thursky KA, Black JF, et al. A prospective comparison of severity scores for identifying patients with severe community acquired pneumonia: reconsidering what is meant by severe pneumonia. Thorax 2006; 61: 419-424.

17 Ewig S, de Roux A, Bauer T, et al. Validation of predictive rules and indices of severity for community acquired pneumonia. Thorax 2004; 59: 421-427.

18 van der Eerden MM, de Graaff CS, Bronsveld W, et al. Prospective evaluation of pneumonia severity index in hospitalised patients with community-acquired pneumonia. Respir Med 2004; 98: 872-878.

19 Aujesky D, Auble TE, Yealy DM, et al. Prospective comparison of three validated prediction rules for prognosis in communityacquired pneumonia. Am J Med 2005; 118: 384-392.

20 Man SY, Lee N, Ip M, et al. Prospective comparison of three predictive rules for assessing severity of community-acquired pneumonia in Hong Kong. Thorax 2007; 62: 348-353.

21 Capelastegui A, Espana PP, Quintana JM, et al. Declining length of stay for pneumonia and post-discharge outcomes. Am J Med 2008; 121: 845-852.

22 Kaplan V, Angus DC, Griffin MF, et al. Hospitalized communityacquired pneumonia in the elderly: age- and sex-related patterns of care and outcome in the United States. Am J Respir Crit Care Med 2002; 165: 766-772.

23 Wilcox MH. Respiratory antibiotic use and Clostridium difficile infection: is it the drugs or is it the doctors? Thorax 2000; 55: 633-634.

24 Mears A, White A, Cookson B, et al. Healthcare-associated infections in acute hospitals which interventions are effective? J Hosp Infect 2009; 71: 307-313.

25 Arnold FW, Ramirez JA, McDonald LC, et al. Hospitalization for community-acquired pneumonia: the pneumonia severity index vs clinical judgement. Chest 2003; 124: 121-124.

26 Aujesky D, McCausland JB, Whittle J, et al. Reasons why emergency department providers do not reply on the pneumonia severity index to determine the initial site of treatment for patients with pneumonia. Clin Infect Dis 2009; 49: e100-e108.

27 Lee RW, Lindstrom ST. A teaching hospital's experience applying the Pneumonia Severity Index and antibiotic guidelines in the management of community-acquired pneumonia. Respirology 2007; 12: 754-758.

28 Espana PP, Capelastegui A, Gorordo I, et al. Development and validation of a clinical prediction rule for severe communityacquired pneumonia. Am J Respir Crit Care Med 2006; 174: 12491256.

29 Charles PG, Wolfe R, Whitby M, et al. SMART-COP: a tool for predicting the need for intensive respiratory or vasopressor support in community-acquired pneumonia. Clin Infec Dis 2008; 47: 375-384.

30 Lyman GH, Kuderer NM. The strengths and limitations of metaanalyses based on aggregate data. BMC Med Res Methodol 2005; 5: 14. 